\title{
Twist - 3 polarized structure functions
}

\author{
Jiro Kodaira ${ }^{a}$ 开 and Kazuhiro Tanaka ${ }^{b}$ \\ ${ }^{a}$ Dept. of Physics, Hiroshima University, Higashi-Hiroshima 739-8526, Japan \\ ${ }^{b}$ Dept. of Physics, Juntendo University, Inba-gun, Chiba 270-1606, Japan
}

We review the nucleon's twist-3 polarized structure functions from the viewpoint of gauge invariant, nonlocal light-cone operators in QCD. We discuss a systematic treatment of the polarized structure functions and the corresponding parton distribution functions. We emphasize unique features of higher twist distributions, and the role of the QCD equations of motion to derive their anomalous dimensions for $Q^{2}$-evolution.

\section{Introduction}

In the last ten years, great progress has been made both theoretically and experimentally in hadron spin physics. Furthermore, in conjunction with new projects like the "RHIC spin project", "polarized HERA", etc., we are now in a position to obtain more information on the spin structure of nucleons.

In the case of spin-dependent processes, it has been known that the twist-3 contributions can be measured as leading effects in certain asymmetries. Since the higher twist effects describe the coherent quark-gluon behavior inside the nucleon, we could obtain information on the correlation of quarks and gluons beyond the (QCD) parton model (leading twist effect).

In this talk, we will concentrate on the twist-3 polarized structure functions [1]. We present a systematic treatment of these functions in terms of gauge invariant, nonlocal light-cone operators, and summarize the recent theoretical progress on the QCD evolutions [2].

\section{Classification of twist-3 structure functions}

To describe a variety of high-energy processes in a universal language, it is desirable to have a definition of parton distribution functions based on the operators in QCD. The traditional approach relies on the operator product expansion (OPE), but it can be applied only to a limited class of processes. This calls for an approach based on the factorization as a generalization of the OPE.

\subsection{Parton distributions}

It is instructive, at first, to consider the deep inelastic scattering (DIS) in free field theories. The result provides a guide to operator definitions of the structure functions (parton distributions). As is well known, the relevant quantity for the DIS is the hadronic tensor:

$$
W_{\mu \nu}=\int \frac{d^{4} z}{2 \pi} e^{-i q \cdot z}\left\langle P S\left|\left[J_{\mu}(0), J_{\nu}(z)\right]\right| P S\right\rangle .
$$

Here $|P S\rangle$ is the hadron state with momentum $P$ and spin $S, q^{\mu}\left(Q^{2}=-q^{2}\right)$ is the momentum transferred to the hadron, and $J_{\mu}$ is the hadron's electromagnetic (weak) current. In the Bjorken limit, the hadronic tensor (11) is governed by the behavior of the current product near the light-cone (light-cone dominance). In the free field theory, the current product can be calculated to be

$$
\left[J_{\mu}(0), J_{\nu}(z)\right]=-\left(\partial^{\alpha} \Delta(z)\right)\left[S_{\mu \alpha \nu \sigma} \mathcal{U}_{V}^{\sigma}(0, z)-i \epsilon_{\mu \alpha \nu \sigma} \mathcal{U}_{A}^{\sigma}(0, z)\right]
$$

\footnotetext{
${ }^{*}$ Talk presented by J. Kodaira at the QCD 99 Euroconference, Montpellier, July 7-13, 1999.

${ }^{\dagger}$ Research supported in part by the Monbusho Grant-in-Aid for Scientific Research No.C(2)09640364.
} 
up to irrelevant terms to the DIS. $\Delta(z)$ is the commutator function, $S_{\mu \alpha \nu \sigma} \equiv g_{\mu \alpha} g_{\nu \sigma}-g_{\mu \nu} g_{\alpha \sigma}+g_{\mu \sigma} g_{\nu \alpha}$, and the nonlocal operators are defined by

$$
\begin{aligned}
& \mathcal{U}_{V}^{\sigma}(0, z) \equiv \bar{\psi}(0) \gamma^{\sigma} \psi(z)-\bar{\psi}(z) \gamma^{\sigma} \psi(0) \\
& \mathcal{U}_{A}^{\sigma}(0, z) \equiv \bar{\psi}(0) \gamma^{\sigma} \gamma^{5} \psi(z)+\bar{\psi}(z) \gamma^{\sigma} \gamma^{5} \psi(0)
\end{aligned}
$$

The singularity of $\Delta(z)$ (or more generally the light cone dominance) selects the integration region with $\eta \sim z_{\perp} \sim 0$ when we write $z^{\mu}$ as $z^{\mu}=\eta p^{\mu}+\lambda w^{\mu}+z_{\perp}^{\mu}$ using two auxiliary light-like vectors $p^{2}=w^{2}=0, p \cdot w=1$. (We take $P^{\mu}=p^{\mu}+\left(M^{2} / 2\right) w^{\mu}$ and $q^{\mu}=-x p^{\mu}+\left(Q^{2} / 2 x+M^{2} x / 2\right) w^{\mu}+\mathcal{O}\left(1 / Q^{2}\right)$ with the hadron mass $M$ and the Bjorken variable $x \equiv Q^{2} /(2 P \cdot q)$.) Therefore, we can expand the matrix element of (22) in powers of the deviation from the light-cone, $z^{\mu}-\lambda w^{\mu}$, and approximate it as

$$
\left\langle P S\left|\mathcal{U}_{V, A}^{\sigma}(0, z)\right| P S\right\rangle \simeq\left\langle P S\left|\mathcal{U}_{V, A}^{\sigma}(0, \lambda w)\right| P S\right\rangle .
$$

By parameterizing the first term of (3) as

$$
\left\langle P S\left|\bar{\psi}(0) \gamma^{\sigma} \psi(\lambda w)\right| P S\right\rangle=2 p^{\sigma} \hat{q}(\lambda)+2 w^{\sigma} M^{2} \hat{f}_{4}(\lambda),
$$

and substituting it into (11), the unpolarized structure function (quark distribution) is defined as the Fourier transform of $\hat{q}(\lambda)$ into the Bjorken- $x$ space,

$$
q(x)=\int \frac{d \lambda}{4 \pi} e^{i \lambda x}\langle P S|\bar{\psi}(0) \not \omega \psi(\lambda w)| P S\rangle .
$$

The second term of (4) is the twist- 4 contribution. Other terms of (2) can be treated similarly. These results yield the factorization into the $c$-number coefficients (short-distance part) and the parton distributions (long-distance part).

In the presence of QCD interaction, the above results are modified in two respects. First, the higher order interactions produce the logarithmic $\left(\ln \left(Q^{2} / \mu^{2}\right)\right)$ corrections to $\Delta(z)$, and correspondingly the parton distribution functions acquire a dependence on the renormalization scale $\mu$. Second, the coupling of the "longitudinal" gluons replaces $\Delta(z)$ as [3]

$$
\Delta(z) \rightarrow \Delta(z)[0, z]
$$

where

$$
[y, z]=\operatorname{Pexp}\left(i g \int_{0}^{1} d t(y-z)_{\mu} A^{\mu}(t y+(1-t) z)\right)
$$

is the path-ordered link operator connecting the points $z_{\mu}$ and $y_{\mu}$ along the straight line. By absorbing this factor, the nonlocal light-cone operators which define the parton distribution functions now preserve gauge invariance.

\subsection{Twist-3 quark distributions}

Now, it is not difficult to define the parton distribution functions in QCD 画. We consider the following quantity involving the nucleon matrix element:

$$
\int_{-\infty}^{+\infty} \frac{d \lambda}{2 \pi} e^{i \lambda x}\langle P S|\bar{\psi}(0)[0, \lambda w] \Gamma \psi(\lambda w)| P S\rangle .
$$

Here $\Gamma$ is a generic Dirac matrix, and the link operator $[0, \lambda w]$ makes the operators gauge invariant. Equation (6) defines the distribution function for a quark with momentum $k \cdot w=x P \cdot w=x$. $\Gamma$ can be any Dirac matrix, depending on which hard process is considered. An important observation made in [5] is that one can generate all quark distribution functions up to twist- 4 by substituting all the possible $\Gamma$. 
By decomposing (6) into independent tensor structures, one finds nine independent quark distribution functions associating with each tensor structure [2]. We list here only the twist-3 polarized distribution functions.

$$
\begin{aligned}
\int \frac{d \lambda}{2 \pi} e^{i \lambda x}\left\langle P S\left|\bar{\psi}(0)[0, \lambda w] \gamma^{\mu} \gamma_{5} \psi(\lambda w)\right| P S\right\rangle & =2 g_{T}\left(x, \mu^{2}\right) S_{\perp}^{\mu}+\cdots \\
\int \frac{d \lambda}{2 \pi} e^{i \lambda x}\left\langle P S\left|\bar{\psi}(0)[0, \lambda w] \sigma^{\mu \nu} i \gamma_{5} \psi(\lambda w)\right| P S\right\rangle & =2 h_{L}\left(x, \mu^{2}\right) M\left(p^{\mu} w^{\nu}-p^{\nu} w^{\mu}\right)(S \cdot w)+\cdots
\end{aligned}
$$

where $S^{\mu}=S_{\|}^{\mu}+S_{\perp}^{\mu}$ with $S_{\|} \cdot w=S \cdot w$.

Three comments are in order [2]. (1) Since the definition of the twist here is based on the simple power counting with respect to $1 / Q$, there is a slight mismatch with the conventional definition as "dimension minus spin" of the relevant operators. Actually both distributions $g_{T}$ and $h_{L}$ contain the contributions from the twist-2 pieces. (2) $g_{T}$ and $h_{L}$ have different chiralities ("even" and "odd"). (3) The light-cone quantization formalism [5, 6] is a conceptually useful approach to the twist counting. In this approach, quark fields are decomposed into "good" and "bad" components. The good component represents an independent degree of freedom; the bad components are not dynamically independent and can be reexpressed by a coherent quark-gluon pair. Therefore, only the twist-2 distributions are literally the "distributions" and correspond to the parton model, and the higher twist distributions are the multiparton (quark-gluon) correlations.

\subsection{Twist-3 gluon distributions}

Our analysis can be extended to the gluon distribution functions. The gauge-invariant definition of the gluon distribution functions is provided by «, 团

$$
\int \frac{d \lambda}{2 \pi} e^{i \lambda x}\left\langle P S\left|w_{\alpha} G^{\alpha \mu}(0)[0, \lambda w] w_{\beta} G^{\beta \nu}(\lambda w)\right| P S\right\rangle=-\frac{x}{2} \mathcal{G}_{3 T}\left(x, \mu^{2}\right) i \epsilon^{\mu \nu \alpha \beta} S_{\perp \alpha} w_{\beta}+\cdots,
$$

where $G^{\mu \nu}$ is the field strength tensor, and we show the twist-3 term explicitly. The gluon distributions mix through renormalization with the flavor singlet chiral-even quark distributions. On the other hand, there exists no gluon distributions that mix with the chiral-odd quark distributions.

\subsection{Twist-3 three-particle distributions}

Coherent many-particle contents of the nucleon are described by multiparton distribution functions. The twist-3 quark-gluon correlation functions are defined as

$$
\int \frac{d \lambda}{2 \pi} \frac{d \zeta}{2 \pi} e^{i \lambda x+i \zeta\left(x^{\prime}-x\right)}\left\langle P S\left|\bar{\psi}(0) \Gamma[0, \zeta w] g G^{\mu \nu}(\zeta w)[\zeta w, \lambda w] \psi(\lambda w)\right| P S\right\rangle
$$

Similarly to the quark distributions, one can define the multiparton distributions by considering possible Dirac matrices for $\Gamma$. The treatment here can be extended to the case of three-gluon correlation functions, which are relevant to the (singlet) quark distribution $g_{T}(x)$ and the gluon distribution $\mathcal{G}_{3 T}(x)$. Although the extension is straightforward, we do not go into the details of the three-gluon nonlocal light-cone operators [8, 9].

\section{QCD evolutions}

The calculation of $Q^{2}$ evolutions for the higher twist terms is generally very complicated due to the presence of multiparton distributions. Although it is possible to generalize the DGLAP approach to the three-body case and calculate its evolution kernel, one is forced to use some particular techniques, e.g. the use of the light-like axial gauge etc. [8, 10] In this talk, we report an approach based on the "local composite operators" in a "covariant" gauge [11]. The advantage of this approach is that all the relevant steps can be worked out based on the standard and familiar field theory techniques. Therefore, 
the calculation is very straightforward and we can assess unambiguously the necessary information for the $Q^{2}$ evolutions.

\subsection{Local operators}

By taking the moment of distribution functions, one can show that there is one-to-one correspondence between the nonlocal and the local operators. The simplest example is provided by the unpolarized twist-2 quark distribution (5):

$$
\begin{aligned}
\int d x x^{n-1} q(x) & =\frac{1}{2} \int d \lambda\left\{\left(\frac{\partial}{i \partial \lambda}\right)^{n-1} \delta(\lambda)\right\}\langle P S|\bar{\psi}(0) \not \omega[0, \lambda w] \psi(\lambda w)| P S\rangle \\
& =\frac{1}{2}\left\langle P S\left|\bar{\psi}(0) \not \omega(i w \cdot D)^{n-1} \psi(0)\right| P S\right\rangle .
\end{aligned}
$$

This equation implies that the moments of the parton distributions are given by the matrix elements of the corresponding gauge-invariant, local composite operators. For the twist-3 distributions, the situation is the same except that one should take into account the "double" moments for the three-body distributions.

\subsection{Operator relations}

The common feature for the higher twist operators is that there appear a set of local operators with the same quantum numbers, the number of which increases with spin (moment), and these operators are not all independent but related through the QCD equation of motion [12, 13.

One of the most convenient method to identify the relevant operators and a relation among them is to utilize the exact operator identities satisfied by the gauge invariant nonlocal operators. In the case of non-singlet part of $g_{T}$, the following operator identity can be obtained by explicit differentiation [3].

$$
\begin{aligned}
& z_{\mu}\left(\frac{\partial}{\partial z_{\mu}} \bar{\psi}(0) \gamma^{\sigma} \gamma_{5}[0, z] \psi(z)-(\sigma \leftrightarrow \mu)\right) \\
& =\int_{0}^{1} d t \bar{\psi}(0)[0, t z] \not z\left\{i \gamma_{5}\left(t-\frac{1}{2}\right) g G^{\sigma \rho}(t z) z_{\rho}-\frac{1}{2} g \tilde{G}^{\sigma \rho}(t z) z_{\rho}\right\}[t z, z] \psi(z) \\
& \quad+2 m_{q} \bar{\psi}(0) \gamma_{5} \sigma^{\sigma \rho} z_{\rho}[0, z] \psi(z) \\
& \quad+\bar{\psi}(0) \gamma_{5} \sigma^{\sigma \rho} z_{\rho}[0, z] E \psi(z)-\psi(0) \overleftarrow{E} \gamma_{5} \sigma^{\sigma \rho} z_{\rho}[0, z] \psi(z)
\end{aligned}
$$

Here $E=i \not D-m_{q}$ is the equation of motion operator and $m_{q}$ represents the quark mass generically. This identity is exact through twist-3. Taylor expanding the above equation around $z_{\mu}=0$, we easily identify the local operators, which contribute to the moment of $g_{T}$, and also the relation among them.

$$
\begin{aligned}
R_{n, F}^{\sigma} & =-i^{n-1} \mathcal{S} \mathcal{A} \mathcal{S} \bar{\psi} \gamma_{5} \gamma^{[\sigma} D^{\left\{\mu_{1}\right]} \cdots D^{\left.\mu_{n-1}\right\}} \psi, \\
R_{n, l}^{\sigma} & =\frac{1}{2 n}\left(V_{l}-V_{n-1-l}+U_{l}+U_{n-1-l}\right), \\
R_{n, m}^{\sigma} & =-i^{n} \mathcal{S} m_{q} \bar{\psi} \gamma^{\sigma} \gamma_{5} D^{\mu_{1}} \cdots D^{\mu_{n-2}} \gamma_{\mu_{n-1}} \psi, \\
R_{n, E}^{\sigma} & =i^{n-2} \frac{n-1}{2 n} \mathcal{S} \bar{\psi}\left[E \gamma^{\sigma} \gamma_{5} D^{\mu_{1}} \cdots D^{\mu_{n-2}} \gamma^{\mu_{n-1}}+\gamma^{\sigma} \gamma_{5} D^{\mu_{1}} \cdots D^{\mu_{n-2}} \gamma^{\mu_{n-1}} E\right] \psi
\end{aligned}
$$

where $\mathcal{S}$ symmetrizes over $\mu_{i}$ and $\mathcal{A}$ antisymmetrizes $\sigma$ and $\mu_{1}$, and

$$
\begin{aligned}
V_{l} & =-i^{n} g \mathcal{S} \bar{\psi} D^{\mu_{1}} \cdots G^{\sigma \mu_{l}} \cdots D^{\mu_{n-2}} \gamma^{\mu_{n-1}} \gamma_{5} \psi, \\
U_{l} & =-i^{n-1} g \mathcal{S} \bar{\psi} D^{\mu_{1}} \cdots \tilde{G}^{\sigma \mu_{l}} \cdots D^{\mu_{n-2}} \gamma^{\mu_{n-1}} \psi .
\end{aligned}
$$

The $R_{n, l}$ is related to the double moment of quark-gluon three-body correlation functions (7) with $\Gamma=\gamma_{\mu}, \gamma_{\mu} \gamma_{5}$. These operators satisfy,

$$
R_{n, F}^{\sigma}=\frac{n-1}{n} R_{n, m}^{\sigma}+\sum_{l=1}^{n-2}(n-1-l) R_{n, l}^{\sigma}+R_{n, E}^{\sigma} .
$$


For the singlet part, the situation becomes much more complicated. We have additional operators that are made of two or three gluon fields, as well as the BRST invariant, gauge variant operators. The explicit expressions for the operators and the derivation of the identity satisfied by them are found in Ref. [9].

For other distributions of twist-3 and higher, we can follow exactly the same procedure. In the case of e.g. $h_{L}$, from the quantity,

$$
\frac{\partial}{\partial z_{\mu}}\left\{\bar{\psi}(0) i \gamma_{5} \sigma_{\mu \nu} z^{\nu}[0, z] \psi(z)\right\}
$$

one can generate towers of identities for the corresponding local operators [2, 5, 14].

\subsection{Calculations}

Since we identify a complete set of operators, the next step is to calculate the anomalous dimensions (renormalization constants) for these operators to derive the $Q^{2}$ evolution. For this task, the following theorem [15] is very helpful. This theorem tells us that in non-abelian gauge theory, three kinds of operators will mix under the renormalization of gauge invariant operators. (I)the gauge invariant operator itself. (II)the BRST invariant operators. (III)the operators which are proportional to the equation of motion (EOM). These EOM operators involve both BRST "invariant" and "variant" ones [11, 16]. Although the physical matrix elements of the BRST invariant and EOM operators vanish [2], it is necessary to consider these operators to complete the renormalization, in other words to obtain the correct renormalization constants for the gauge invariant operators.

At the lowest twist level, the above complicated operator mixing does not come into play, because there exists neither an EOM nor BRST invariant operator of twist-2. The EOM as well as the BRST invariant operators always have smaller spin by at least one unit than the possible highest spin operators of the same dimension. At the higher twist $(\geq 3)$ level, both EOM and BRST invariant operators participate in the renormalization mixing with the gauge invariant operators. This is a characteristic feature of the renormalization for the higher twist operators.

The explicit calculations at the one-loop level appear in Refs. 11, 9, 17] for $g_{T}$ and in Ref. 14] for $h_{L}$.

\section{Conclusions}

We have surveyed the twist-3 polarized structure functions from a viewpoint of gauge invariant nonlocal operators. One of the key points of our approach is that we preserved maximal (gauge (BRST) and Lorentz) symmetries of the theory at every step of investigation. Another point is use of the one-to-one correspondence between the nonlocal and the local operator approaches to investigate the $Q^{2}$-evolution. We have clarified the role of the QCD equations of motion to reveal the interrelation between the different twist-3 distributions and to derive their $Q^{2}$-evolution.

The polarized twist-3 distribution functions $g_{T}$ and $h_{L}$ are measurable as leading effects and are of special interest. However, the phenomenology of twist-3 distributions is not as straightforward as that of twist-2 distributions due to the fact that the twist-3 distributions are essentially three-particle correlations: We need to determine many parameters to make precise predictions. In this respect, it is pointed out that a drastic and universal simplification of all twist-3 distributions occurs in the $N_{c} \rightarrow \infty$ limit [18]. It would be a good news to solve the above problem for all practical purposes.

We hope that various kinds of new experiments and more detailed theoretical investigations will be able to clarify not only perturbative but also nonperturbative aspects of QCD related to hadron spin physics.

\section{References}


[1] A. V. Belitsky, in the XXXI PNPI Winter School on Nuclear and Particle Physics, (St. Petersburg, 1997) p. 369, hep-ph/9703432; hep-ph/9907420, and references therein.

[2] J. Kodaira and K. Tanaka, Prog. Theor. Phys. 101 (1999) 191.

[3] I. I. Balitsky and V. M. Braun, Nucl. Phys. B311 (1988/89) 541.

[4] J. C. Collins and D. E. Soper, Nucl. Phys. B194 (1982) 445.

[5] R. L. Jaffe and X. Ji, Phys. Rev. Lett. 67 (1991) 552. Nucl. Phys. B375 (1992) 527.

[6] J. B. Kogut and D. E. Soper, Phys. Rev. D1 (1970) 2901.

[7] A. V. Manohar, Phys. Rev. Lett. 65 (1990) 2511; Phys. Rev. Lett. 66 (1991) 289.

[8] A. P. Bukhvostov, E. A. Kuraev and L. N. Lipatov, Sov. J. Nucl. Phys. 38 (1983) 263; 39 (1984) 121. JETP Letters 37 (1984) 483. Sov. Phys. JETP 60 (1984) 22.

[9] J. Kodaira, T. Nasuno, H. Tochimura, K. Tanaka and Y. Yasui, Prog. Theor. Phys. 99 (1998) 315; in Proc. of 2nd Topical Workshop on Deep Inelastic Scattering off Polarized Targets: Theory Meets Experiment (DESY-Zeuthen, 1997), DESY 97-200, p. 210.

[10] B. Geyer, D. Müller and D. Robaschik, Nucl. Phys. Proc. Suppl. 51C (1996) 106. D. Müller, Phys. Lett. B407 (1997) 314.

[11] J. Kodaira, Y. Yasui and T. Uematsu, Phys. Lett. B344 (1995) 348.

J. Kodaira, Y. Yasui, K. Tanaka and T. Uematsu, Phys. Lett. B387 (1996) 855.

[12] H. D. Politzer, Nucl. Phys. B172 (1980) 349.

[13] E. V. Shuryak and A. I. Vainshtein, Nucl. Phys. B199 (1982) 951; Nucl. Phys. B201 (1982) 141.

[14] Y. Koike and K. Tanaka, Phys. Rev. D51 (1995), 6125.

[15] S.D. Joglekar and B.W. Lee, Ann. of Phys. 97 (1976) 160.

[16] H. Kawamura, T. Uematsu, J. Kodaira and Y. Yasui, Mod. Phys. Lett. A12 (1997) 135.

[17] Y. Koike, N. Nishiyama and K. Tanaka, Phys. Lett. B437 (1998) 153.

[18] A. Ali, V.M. Braun and G. Hiller, Phys. Lett. B266 (1991) 117.

I. I. Balitsky, V. M. Braun, Y. Koike and K. Tanaka, Phys. Rev. Lett. 77 (1996) 3078.

K. Sasaki, Phys. Rev. D58 (1998) 094007.

X. Ji and J. Osborne, Euro. Phys. J. C9 (1999) 487. 